\title{
INFRARED SPECTROSCOPY: A TOOL FOR DETERMINATION OF THE DEGREE OF CONVERSION IN DENTAL COMPOSITES
}

\author{
Luciene Gonçalves Palmeira MORAES ${ }^{1}$, Renata Sanches Ferreira ROCHA ${ }^{1}$, Lívia Maluf MENEGAZZO ${ }^{1}$, \\ Eudes Borges de ARAÚJO ${ }^{2}$, Keizo YUKIMITU², João Carlos Silos MORAES ${ }^{3}$
}

\begin{abstract}
1- DDS, MSc, Department of Physics and Chemistry, São Paulo State University, Ilha Solteira, SP, Brazil.
2- DDS, MSc, PhD, Associate Professor, Department of Physics and Chemistry, São Paulo State University, Ilha Solteira, SP, Brazil.

3- DDS, MSc, PhD, Full Professor, Department of Physics and Chemistry, São Paulo State University, Ilha Solteira, SP, Brazil.
\end{abstract}

Corresponding address: Prof. Dr. João Carlos Silos Moraes - Departamento de Física e Química - UNESP - Av. Brasil Centro 56 - Caixa Postal 31 - 15385-000 - Ilha Solteira, SP, Brasil - joca@dfq.feis.unesp.br - Phone: + 5518 3743-1029 - Fax: + 5518 3742-4868

Received: September 04, 2007 - Modification: November 19, 2007 - Accepted: December 13, 2007

\begin{abstract}
$I_{\mathrm{n}}$

nfrared spectroscopy is one of the most widely used techniques for measurement of conversion degree in dental composites. However, to obtain good quality spectra and quantitative analysis from spectral data, appropriate expertise and knowledge of the technique are mandatory. This paper presents important details to use infrared spectroscopy for determination of the conversion degree.
\end{abstract}

Key words: Dental composites. Degree of conversion. Infrared spectroscopy. FTIR.

\section{INTRODUCTION}

The physical and mechanical properties of photo-cured composites are directly influenced by the level of conversion attained during polymerization. The degree of conversion (DC) is determined by the proportion of the remaining concentration of the aliphatic $\mathrm{C}=\mathrm{C}$ double bonds in a cured sample relative to the total number of $\mathrm{C}=\mathrm{C}$ bonds in the uncured material. Typical composites used in dental restorative applications are composed of at least two dimethacrylate monomers. The cured composites based on monomers with high molecular weight, as the Bis-GMA or UEDMA, exhibit a considerable number of remaining double bonds $\mathrm{s}^{3,13}$. The limited conversion found in many network polymers is due to restricted mobility of radical chain ends, pendant methacrylate and monomer imposed at high crosslink density ${ }^{5}$. In general, the photoactivated dental composites reach a DC ranging from 43 to $75 \%$, basically depending on the composite composition, irradiation intensity and exposure time $\mathrm{s}^{5,11}$.

Techniques such as Fourier transform infrared (FTIR) spectroscopy $^{3,13,14}$, RAMAN ${ }^{4,12}$, EPR $^{15}$ (electron paramagnetic resonance), $\mathrm{NMR}^{9}$ (nuclear magnetic resonance), $\mathrm{DSC}^{1}$ (differential scanning calorimetry) and DTA $^{6,8}$ (differential thermal analysis) have been used to determine the DC. Among these, FTIR is the most frequently used technique.
The use of FTIR spectroscopy requires certain precautions during sample preparation and method performance in order to obtain appropriate spectral data that will provide an accurate determination of the conversion degree. Therefore, this paper presents the foundations and experimental details that can aid researchers interested in using this technique with ultimate outcomes.

\section{FTIR SPECTROSCOPY}

FTIR spectroscopy is a widely used technique for investigating materials in the gaseous, liquid or solid phase. It is based on the interaction between electromagnetic radiation and natural vibrations of the chemical bonds among atoms that compose the matter. Not all possible vibrations within a molecule will result in an absorption band in the infrared (IR) region. For a material to absorb radiation in the infrared region, two conditions should be fulfilled: (1) there must be coincidence (resonance) among the frequencies of the infrared radiation and molecular vibration; (2) the natural vibration must cause change in the dipole moment during vibration.

The vibration frequencies of a chemical bond depend on the stiffness of this bond (characterized by a proportionality constant termed the force constant) and the masses of the atoms at each end of the bond. There are two types of molecular vibrations: one that changes the bond length (stretching) and other that changes the bond angle 
(bending).

The position of absorption bands in the spectra are presented as wavelength $(\lambda)$, either as meters or submultiples of a meter. In the infrared region, the commonly used unit is the wavenumber $(\bar{v})$, which is expressed in $\mathrm{cm}^{-1}$, because it is directly proportional to energy $(E)$ and frequency $(v)$ of radiation, according to the equation:

$$
E=h v=\frac{h c}{\lambda}=h c \bar{v}
$$

where $h$ is the Planck's constant and $c$ is the speed of light in vacuum.

The band intensity can be expressed in transmittance or absorbance. Transmittance $(T)$ is defined as the ratio between intensities of the transmitted $(I)$ and incident $\left(I_{o}\right)$ beams. On the other hand, absorbance $(A)$ is the logarithm (base 10) of the reciprocal of the transmittance:

$$
A=\log _{10}\left(\frac{1}{T}\right)=\log _{10}\left(\frac{I_{0}}{I}\right)
$$

The transmitted radiant energy depends on the thickness $(x)$ and absorption coefficient $(\alpha)$ of the sample, according to the equation:

$$
I=I_{0} e^{\alpha x}
$$

Infrared is often divided into three spectral regions, i.e., the near (NIR - from 4,000 to approximately $\left.14,000 \mathrm{~cm}^{-1}\right)$, mid (MIR - from 400 to $4,000 \mathrm{~cm}^{-1}$ ) and far-infrared (FIR - from approximately 25 to $400 \mathrm{~cm}^{-1}$ ). In the contemporary spectrometers, it is possible to work in these three spectral regions, thus it is necessary to change some of their optical components in order to shift to another spectral region.

The main components of a contemporary spectrometer are: IR source, Michelson interferometer and IR detector. The more stable source of radiation, emitting in the three IR regions is the Ever-Glo ceramic. By using the appropriate beamsplitter (interferometer component) and IR detector in combination with this source, it is possible to provide energy for the spectral range from 7,400 to $50 \mathrm{~cm}^{-1}$. The Michelson interferometer basically consists of two perpendicular plane mirrors (one fixed and the other movable) and a beamsplitter. The function of an interferometer is to split a beam of light into two beams and to introduce an optical path difference between them. As a consequence, the path difference creates the condition for that interference to take place when the beams recombine at the beamsplitter. The intensity variations of the beam emerging from the interferometer are monitored as a function of path difference by the detector. The light intensity versus the optical path difference is called an interferogram. The Fourier transform is the mathematic tool used to convert the interferogram into a spectrum, which is done by a computer.

To obtain a spectrum, one must first record a background (bg) spectrum, which contains the absorptions of molecules present in the air. Thereafter, another spectrum is recorded with the sample in place. This spectrum contains absorptions of both the sample and the atmospheric molecules. Dividing the sample spectrum by the background spectrum removes all absorptions contained in the bg so that the absorption peaks in the final spectrum are due solely to the sample.

When a sample absorbs some of the infrared radiation, the intensity of the transmitted and reflected radiation is reduced. Regardless of the spectral region one is working on, the spectra can be obtained by transmission or reflection method. The transmission method is the oldest and most commonly used currently. In this arrangement, the detector measures the intensity of the infrared radiation passing through the sample (transmitted radiation). In the reflection method, the detector measures the infrared radiation reflected by the sample. In current days, there are different reflectance methods, such as the attenuated total reflectance (ATR), diffuse and specular reflectance. For each method there is a proper accessory.

There are different forms to prepare the sample to be analyzed by the transmission method. The choice for a method strongly depends on the nature of the sample to be analyzed. For example, for solid samples, mixing a small amount of sample with a dry potassium bromide $(\mathrm{KBr})$ powder is usually necessary. $\mathrm{KBr}$ is completely transparent in the MIR region. For liquid samples, there are proper cells with a fixed path length, sealed or not. Independently of the cell to be used, the window material must be transparent for the incident infrared radiation.

\section{DETERMINATION OF THE CONVERSION DEGREE}

To determine DC in composites based on methacrylates, two spectral infrared regions can be used; NIR or MIR. In the MIR region, $\mathrm{DC}$ is determined by measuring the intensity (or area) decrease of the methacrylate $(\mathrm{C}=\mathrm{C})$ stretch absorption band at $1,638 \mathrm{~cm}^{-1}$ as the methacrylate monomer is converted to polymer. In the NIR region, there are two aliphatic bands that can be used, one at $6,165 \mathrm{~cm}^{-1}$ (overtone $=\mathrm{CH}_{2}$ ) and the other at $4,743 \mathrm{~cm}^{-1}$ (combination of $=\mathrm{CH}_{2}$ bands). Regardless of the spectral region to be used, determination of DC is facilitated when the material presents a stable absorption band, whose intensity is unaltered during polymerization process. This band is used as an internal standard of normalization. In this case, it is unnecessary to account the sample thickness. Generally, composites based on methacrylate monomers present aromatic bands at 1,583, 1,608 , and $4,623 \mathrm{~cm}^{-1}$, which can be used as internal standards ${ }^{14}$. The band at $4,623 \mathrm{~cm}^{-1}$ is usually not recommended because, depending on resin composition, it can to undergo slight variation in its intensity ${ }^{14}$, introducing error in the DC determination. Therefore, it is important to always verify the stability of this band before using it. In the absence of an isolated stable band, a calibration curve for $\mathrm{C}=\mathrm{C}$ concentration of monomer that composes the composite $^{2,7,10}$ is necessary.

\section{Using internal standard of normalization}

For composites having internal standard, the percentage of unreacted aliphatic $\mathrm{C}=\mathrm{C}$ bonds remaining throughout the polymerization reaction is obtained by the equation: 
$(\% C=C)=\frac{[\text { Abs (aliphatic) } / \text { Abs (aromatic) }]_{\text {polymer }}}{[\text { Abs (aliphatic) } / \text { Abs (aromatic) }]_{\text {monomer }}} \times 100$

where $A b s$ can be the height or the area of absorption band. $\mathrm{DC}$ is determined by subtracting the residual percentage of aliphatic $\mathrm{C}=\mathrm{C}$ from $100 \%(D C \%=100-(\% C=C))$.

The MIR or NIR spectral region should be used with caution to evaluate the quantity of remaining double bonds. In the MIR, the region where the $\mathrm{C}=\mathrm{C}$ aromatic and aliphatic absorptions are located lies embedded in the water region (Figure 1b). Same behavior is not observed in the NIR region (Figure 1a). Thus, any difference in water vapor concentration between background and sample spectra may raise deformations in the final spectrum (Figure 2). This becomes a problem when reliable quantitative data are expected. Figure 2 distinctly illustrates the deformations caused in the spectrum, mainly in the band peak at $1,638 \mathrm{~cm}^{-1}$ (vertical arrow). To solve this problem, the spectrometer should be purged with inert gas (dry nitrogen is recommended).

Figures 3 and 4 show absorption spectra of TPH composite (Table 1) in the MIR and NIR region, respectively. Here, the aliphatic and aromatic absorption bands are highlighted, before and after the polymerization, which are used to evaluate DC. It can be observed that the intensities of the bands used as internal standard do not change during the polymerization process.

\section{Using standard or calibration curve}

Depending on the dental composite it is not always possible to localize a stable band to use as internal standard. For example, in the NIR and MIR spectra of the Acron MC and Classic composites (information in Table 1) it was not possible to localize a stable and resolved band that could be used as internal standard. In such cases, it is necessary

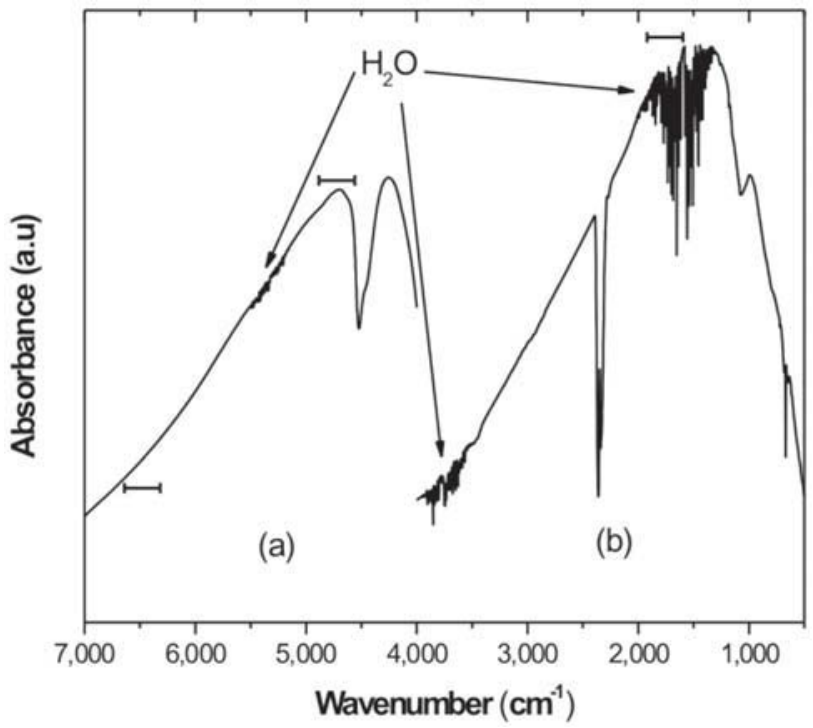

FIGURE 1- Background spectrum in NIR (a) and MIR(b) spectral regions. The horizontal bars indicate where the absorption bands used to determine the conversion degree are localized (a.u.= arbitrary units)

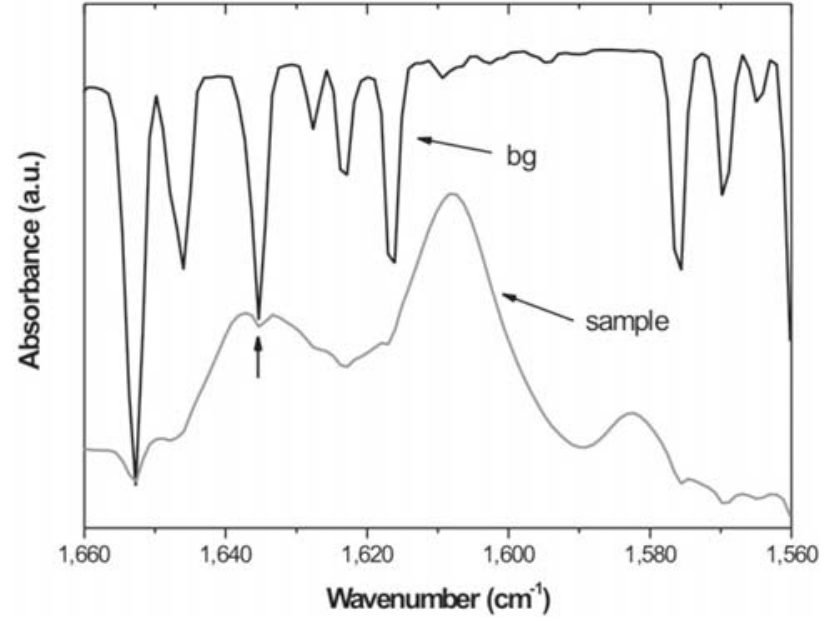

FIGURE 2- Deformed absorption spectrum of dental composite sample (bottom) and the background (bg) spectrum (top) showing the presence of water absorptions (a.u.= arbitrary units)

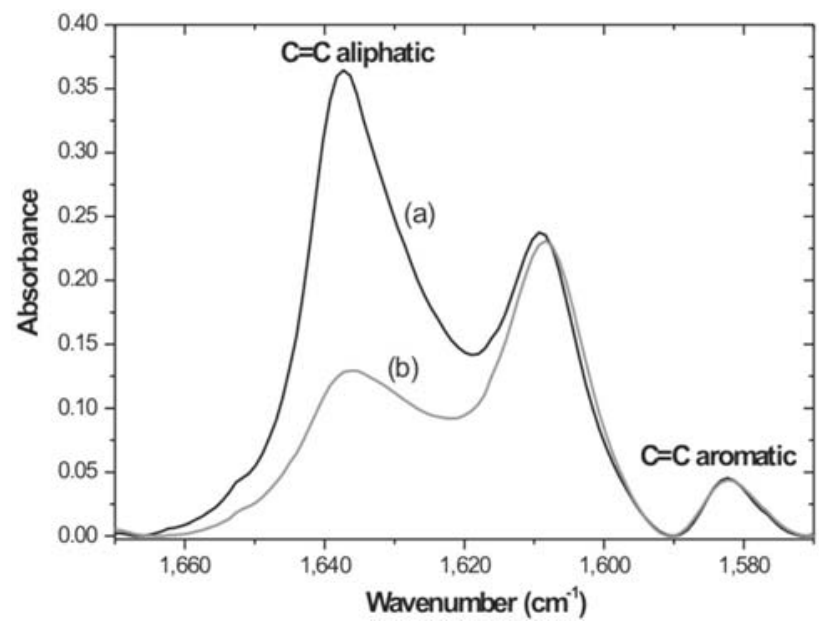

FIGURE 3- TPH absorption bands in the MIR region before (a) and after (b) polymerization

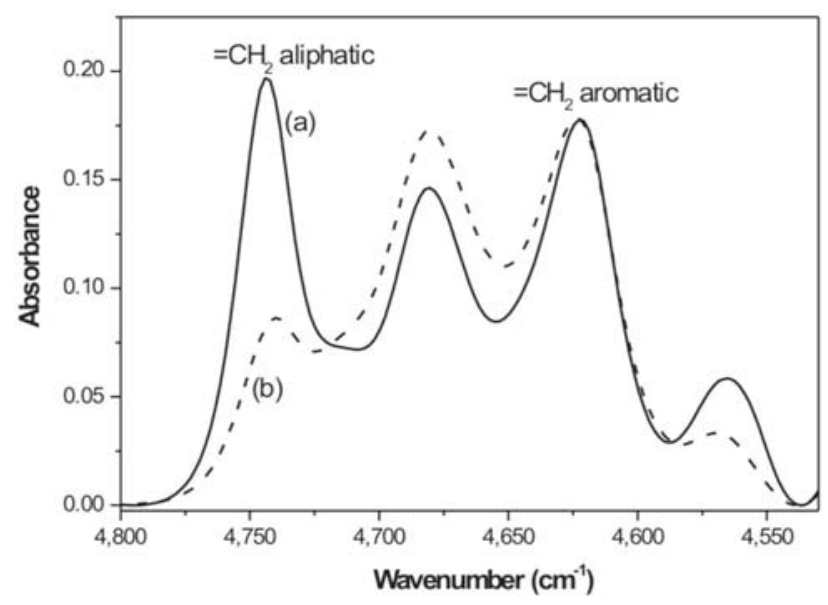

FIGURE 4- TPH absorption bands in the NIR region before (a) and after (b) polymerization 
TABLE 1- Information about the composites used in this study

\begin{tabular}{|c|c|c|}
\hline Material & Manufacturer & Monomers \\
\hline TPH Spectrum & Dentsply Ind. Com. Ltda., Petrópolis, RJ, Brazil & Urethane modified Bis-GMA \\
\hline \multirow[t]{2}{*}{ ACAcron } & GC Dental Ind. Corp., Tokyo, Japan & Methyl methacrylate (95\%) \\
\hline & & Ethyleneglicol dimethacrylate $(5 \%)$ \\
\hline \multirow[t]{2}{*}{ Classic } & Clássico Artigos Odontológios Ltda, SP, Brazil & Methyl methacrylate \\
\hline & & Topanol and ethyleneglicol dimethacrylate \\
\hline
\end{tabular}

to build a calibration curve. These resins are composed of two parts, with one of them in powder (polymer) form and the other one liquid (monomer). For these two composites and other methacrylate-based resins, one can use the method developed by Loshaek and Fox ${ }^{7}$ (1953), which was verified by Rueggeberg ${ }^{10}$ (1994) and Bartoloni, et al. ${ }^{2}(2000)$. This method is based on the assumption that the methacrylate group $\left(\mathrm{H}_{2} \mathrm{C}=\mathrm{C}\left(\mathrm{CH}_{3}\right) \mathrm{C}=\mathrm{O}\right)$ can be used as a gravimetric form of concentration measurements. The IR absorption peak height for the aliphatic $\mathrm{C}=\mathrm{C}$ group divided by the product of the path length and the weight percent methacrylate group can be considered as an optical constant $(\mathrm{K})$. Monomer conversion degree is expressed as a change in weight percent of the methacrylate groups (WPMG) remaining after polymerization, when compared to the amount found prior to curing. Rueggeberg ${ }^{10}$ (1994) worked with different methacrylate-based resins and obtained a mean $\mathrm{K}$ value equal to 0.64 .

The calibration curve is obtained by diluting the monomer of composite in spectrophotometric grade hexane in different concentrations. The curve establishes the relationship between IR absorption peak height and $\mathrm{C}=\mathrm{C}$ concentration (moles $\mathrm{C}=\mathrm{C} / \mathrm{mL}$ ). The number of moles of $\mathrm{C}=\mathrm{C}$ per milliliter of each standard solution is determined by dividing the number of moles of methacrylate units present in the undiluted monomer volume by the volume of the solution (in $\mathrm{mL}$ ). The number of moles of methacrylate units (= number of moles of $\mathrm{C}=\mathrm{C}$ ) is obtained by dividing the undiluted monomer mass by the molecular weight of a methacrylate unit $(69,081)$.

As an example, for ACRON MC composite four different dilutions were prepared and the NIR infrared spectra were obtained using a transmission micro-liquid cell with $\mathrm{KBr}$ windows at a path length of $0.528 \mathrm{~mm}$. The spectra (Figure 5) were obtained from 64 scans at a resolution of 4 $\mathrm{cm}^{-1}$ on a FTIR spectrometer (Nexus 670 of Nicolet). The spectral region used was from 6,050 to $6,300 \mathrm{~cm}^{-1}$, where the $=\mathrm{CH}_{2}$ overtone band at $6,165 \mathrm{~cm}^{-1}$ is localized.

The mathematical relationship between absorbance and $\mathrm{C}=\mathrm{C}$ concentration is obtained from the calibration curve, which enables determining the $\mathrm{C}=\mathrm{C}$ molar concentration in the undiluted monomer. Figure 6 shows, as an example, the calibration curve obtained for ACRON MC

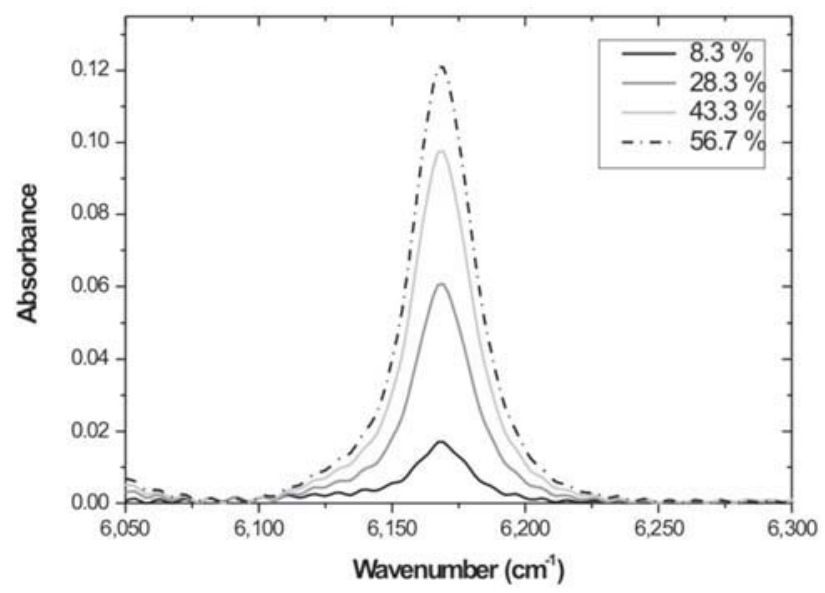

FIGURE 5- Spectra with corrected baseline of a diluted (with hexane) monomer of ACRON MC composite

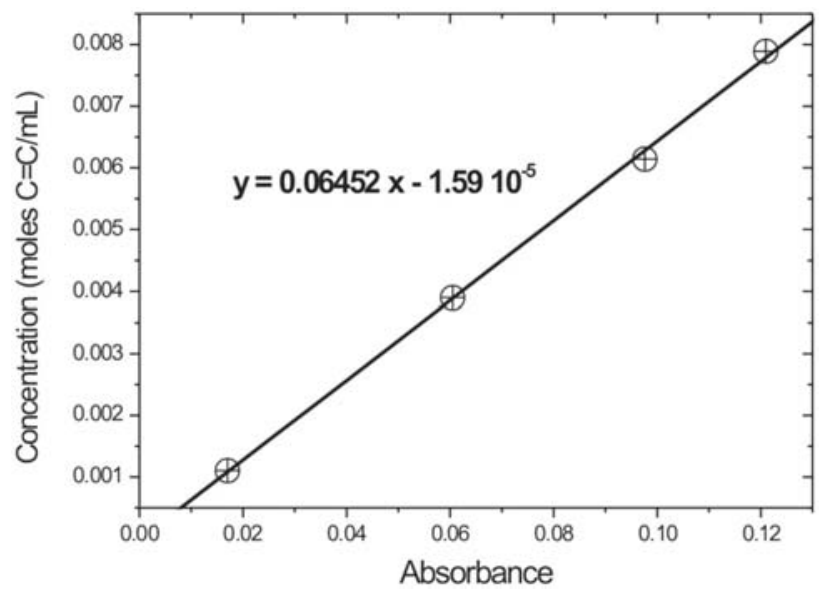

FIGURE 6- Standard or calibration curve obtained with a monomer of ACRON MC composite

composite.

With the curve, it is now possible to study the DC of composites, which is defined as follows:

$$
C D \%=\left[1-\frac{W P M G_{\text {cur }}}{W P M G_{\text {uncur }}}\right] \times 100
$$


where $W P M G_{c u r}$ and $W P M G_{\text {uncur }}$ are weight percentage of methacrylate groups in cured and uncured composite, respectively.

To determine $W P M G_{u n c}$, a spectrum of undiluted monomer should be obtained. However, in order to use the calibration curve it is necessary to perform the measurement using the liquid cell with the same path length as the one used to build the curve. The $\mathrm{C}=\mathrm{C}$ concentration (moles $\mathrm{C}=\mathrm{C}$ / $\mathrm{mL}$ ) present in the undiluted monomer is determined from the mathematical relationship. Therefore, $W P M G_{u n c}$ is calculated by multiplying the $\mathrm{C}=\mathrm{C}$ molar concentration by the molecular weight of a methacrylate unit $(69,081)$ and dividing the result by the monomer density $(\mathrm{g} / \mathrm{mL})$.

$W P M G_{c u r}$ is determined from IR spectrum of cured resin film. For ACRON MC composite, $0.5 \mathrm{~mm}$-thick samples are sufficient to obtain a good spectrum in the NIR region. From the spectrum, absorbance peak height is then determined. Dividing this value by the product between sample thickness and $\mathrm{K}(0.64)$, one can obtain $W P M G_{\text {cur }}$.

\section{CONCLUSIONS}

Although IR spectroscopy is a widely utilized technique, scrutiny is mandatory when choosing the best methodology to obtain the spectra that will enable a reliable quantitative analysis. The study of conversion degree in dental composites by FTIR technique provides a better understanding of these materials, which is expected to optimize the polymerization process. This will result in improved dental restorations with aggregated higher quality and durability.

\section{REFERENCES}

1- Antonucci JM, Toth EE. Extent of polymerization of dental resins by differential scanning calorimetry. J Dent Res. 1983;62:1215 .

2- Bartoloni DF, Murchison DF, Wofford DT, Sarkar NK. Degree of conversion in denture base materials for varied polymerization techniques. J Oral Rehabil. 2000;27:488-93.

3- Chung KH, Greener HE. Degree of conversion of seven visible light-cured posterior composites. J Oral Rehabil. 1988;15:555-60.

4- Gauthier MA, Stangel I, Ellis TH, Zhu XX. A new method for quantifying the intensity of the $\mathrm{C}=\mathrm{C}$ band of dimethacrylate dental monomers in their FTIR and Raman spectra. Biomaterials. $2005 ; 26: 6440-8$.

5- Halvorson RH, Erickson RL, Davidson CL. The effect of filler and silane content on conversion of resin-based composite. Dent Mater. 2003;19:327-33.

6- Imazato S, McCabe JF, Tarumi H, Ehara A, Ebisu S. Degree of conversion of composites measured by DTA and FTIR. Dent Mater. $2001 ; 17: 178-83$.

7- Loshaek S, Fox TG. Cross-linked polymers: I. Factors influencing the efficiency of cross-linking in copolymers of methyl methacrylate and glycol dimethacrylates. J Am Chem Soc. 1953;75:3544-50.
8- McCabe JF. Cure performance of light-activated composites by differential thermal analysis (DTA). Dent Mater. 1985;1:231-4.

9- Morgan DR, Kalachandra S, Shobha HK, Gunduz N, Stejskal EO. Analysis of a dimethacrylate copolymer (BisGMA and TEGDMA) network by DSC and 13C solution and solid-state NMR spectroscopy. Biomaterials. 2000;21:1897-903.

10- Rueggeberg FA. Determination of resin cure using infrared analysis without an internal standard. Dent Mater. 1994;10:282-6.

11 - Ruyter IE, Oyased H. Conversion in different depths of ultraviolet and visible light-actived composite materials. Acta Odont Scand. 1982;40:179-92.

12- Shin WS, Li XF, Schwartz B, Wunder SL, Baran GR. Determination of the degree of cure of dental resins using Raman and FT-Raman spectroscopy. Dent Mater. 1993;9:317-24.

13- Silikas N, Eliades G, Watts DC. Light intensity effects on resincomposite degree of conversion and shrinkage strain. Dent Mater. 2000;16:292-6.

14- Stansbury JW, Dickens SH. Determination of double bond conversion in dental resins by near infrared spectroscopy. Dent Mater. 2001;17:71-9.

15- Sustercic D, Cevc P, Funduk N, Pintar MM. Determination of curing time in visible-light-cured composite resins of different thickness by electron paramagnetic resonance. J Mater Sci Mater Med. 1997;8:507-10. 